\title{
¿Cuál es la concepción de ciencia que se refleja cuando los maestros de ciencias naturales organizan los contenidos a enseñar?
}

\author{
What is the concept of science show that when the teachers of natural sciences \\ organized content to teach?
}

\author{
Lucila Medina de Rivas ${ }^{1}$ y Luz Stella Mejía Aristizábal ${ }^{2}$
}

${ }^{1}$ Magister en Educación. ${ }^{2}$ Magister en Educación. Estudiante de doctorado en Educación. Miembro del Grupo de Investigación Estudios Culturales sobre la Ciencia y su Enseñanza. ECCE. Universidad de Antioquia. Centro Formativo de Antioquia. lucy.medinap@gmail.com, luzes1stel@gmail.com

\section{Resumen}

Esta investigación tiene como objeto determinar las ideas alternativas sobre la concepción de ciencia maestros en ejercicio y al mismo tiempo estudiantes de un programa de educación avanzada, reflejada en la forma como organizan los contenidos de la disciplina que enseñan. Este estudio cualitativo buscar determinar si las concepciones alternativas han sufrido cambios después de que los estudiantes han pasado por programas de formación inicial y avanzada de maestros.

\section{Palabras claves}

Maestros, Formación continua, Concepciones Alternativas, Ciencia, Estructuración de contenidos.

\section{Abstract}

This research aims to identify alternative ideas on the design of masters in exercise science while students in a program of advanced education, as reflected in the form organizes the contents of the discipline they teach. This qualitative study seeks to determine whether the alternative conceptions have changed after the students have gone through training programs and advanced teachers.

\section{Key words}

Teacher training, alternative conceptions, Science, structuring of content.

\section{Introducción}

En los diseños curriculares y espećficamente en la forma como se estructuran los contenidos a enseñar es necesaria la participación activa del maestro; pues sus reflexiones sobre el proceso de enseñanza son fundamentales a la hora de generar propuestas que contribuyan con una mejor comprensión de las ciencias naturales. Esta situación replantea el papel del maestro de ciencias y de los programas de formación inicial y avanzada, ya que hasta el momento estos programas no han incidido en la forma como se estructuran los contenidos a enseñar.

Es necesario tener presente que la forma como se estructure el contenido es un punto clave en la enseñanza, es un proceso que requiere de un análisis del contenido de la disciplina, de su lógica misma y de las dificultades de aprendizaje de los estudiantes. Tal reflexión debe posibilitar una intervención en el aula que tenga en cuenta no sólo las estrategias de enseñanza, sino también los objetivos y actividades evaluativas enmarcadas dentro de una concepción de ciencia diferente que posibilite un curníaulo procesual, abierto y flexible. (Sánchez, G., 2000, p. 423) 
Algunas investigaciones sobre la forma como los profesores planifican los contenidos, afirman que la atención del profesor se dirige principalmente al contenido disaiplinar (Cark y Peterson, 1986) (Bellón et al., 1992, Brickhouse, 1993; Sánchez y Valcárcel, 1999) Cítados en: (Sánchez, G., 2000, p. 424)

Otras se dirigen a la importancia que le dan los docentes a la estructura de los contenidos que se presenta en los libros textos. (Bellón et al., 1992)(424) Adicionalmente a esto, las investigaciones sobre las concepciones de ciencia de los docentes en formación inicial y avanzada muestran que son pocos los cambios que suelen darse en este sentido a pesar de que el docente halla pasado por dichos programas de formación. Los siguientes aspectos son algunos de los supuestos básicos que nos guiarán en el análisis de las ideas alternativas de los estudiantes de educación avanzada a la hora de estructurar los contenidos a enseñar:

> El docente que enseña una disciplina científica tiene un marco teórico amplio, no siempre explícito, darificado o consciente, que da forma a las ideas sobre ciencia presentes en sus dases (es decir, de qué habla cuando habla de ciencia).

$>$ Ese docente también efectúa una transposición didáctica de los conocimientos científicos (para convertirlos en contenido a enseñar) que también puede llegar a ser implíata, o por lo menos, no cuestionada o vigilada epistemológicamente.

Consideramos las anteriores afirmaciones como directrices a la hora de analizar las formas como los estudiantes de formación avanzada organizan los contenidos desde una mirada epistemológica. De ahí que, sirvan de base para conocer las ideas o concepciones altemativas que presenta y que constituyen uno de los aspectos didácticos básicos para comprender y evaluar los procesos de enseñanza.

\section{Descripción del marco conceptual}

Primero que todo intentaremos esdarecer nuestras propias ideas sobre "ciencia" y su didáctica. Comenzaremos esta investigación bajo el supuesto de que los maestros de las disciplinas científicas (Física y química) no siempre tienen daras sus concepciones sobre la ciencia y sobre cómo deben enseñarla.

Resaltamos como importante antes de introducimos en el análisis de las ideas alternativas, el supervisar nuestras propias concepciones de ciencia. Este análisis lo haremos, a partir de los aportes dados desde la epistemología de la ciencia y desde la didáctica de la ciencia enfatizando en aquellos aspectos que tengan relación directa con la problemática planteada en la investigación.

\section{Estructuración de los contenidos: Subject Matter Structure (SMS)}

El concepto de estructuración de los contenidos pertenece espećficamente al campo educativo e instruccional, en un sentido tradicional se refiere al análisis de una materia o contenidos temáticos en términos lógicos o epistemológicos, tal y como se presenta en la disciplina, sin atender a los componentes 0 análisis psicológicos que exige su aprendizaje (Resnick, 1976, Merril, 1987, Jonassen, Hannum y Tessmer,1989) Citados en: (Castejón, J.L., 2002, p. 1)

Es necesario tener presente que desde la orientación cognitiva es importante conocer que una descripción psicológica de los contenidos se hace necesaria, pues la organización lógica de la misma no corresponde con la organización psicológica del sujeto que aprende. Entendiéndose entonces son disciplinas diferentes pero que se hace posible extender un puente entre ellas: la epistemología y la sicología. 
Los fines de los SMS, son de orden epistemológico. Se refieren al origen, a la estructura, a los métodos y a la validez del saber. El objetivo es que al término de estos estudios el profesor logre adoptar una filosofía del conocimiento que sea producto de la reflexión. La importancia se reviste en la determinación de aquellos contenidos que serán relevantes para ser enseñados; su organización y estructuración.

Fuente epistemológica

La idea de cómo se genera el conocimiento cient́fico, esta relacionado con la idea de cómo aprenden las personas, tal relación puede dar origen a estrategias de enseñanza. Desde este punto de vista se pueden analizar los diferentes modelos de enseñanza y aprendizaje, que los profesores de ciencias siguen en el aula. No siendo siempre conscientes del origen epistemológico y psicológico de sus modelos de ciencia.

Dentro de las visiones de ciencia de los profesores encontramos aquella concepción de la ciencia mirada como un conjunto cerrado o definitivo de conceptos, fenómenos o eventos, en donde se asume la ciencia como acabada y sin oportunidad de cambio; o también es posible mirarla como; un continuo proceso de elaboración, susceptible a cambios, una ciencia en construcción que trata de dar respuesta a los problemas científicos que la humanidad plantea. (Murillo, B., 1998, p. 16)

En otra visión, la ciencia se puede concebir como una acumulación de conocimientos que va creciendo en forma vertical o lineal, donde cada cientŕfico va agregando un conocimiento más avanzado a la estructura de conocimiento ya existente. Aquí el conocimiento es basado en múltiples rectificaciones obtenidas a través de la superación de obstáculos y rupturas. Es esta perspectiva; el conocimiento puede entenderse como una construcción personal, la cual responde a reglas que hacen parte del llamado método cientúfico, o se puede ver como una construcción social e histórica condicionada por el pensamiento y el contexto de la época. (p. 17).

A continuación realizamos un análisis con una base epistemológica que nos permita tener daridad con respecto las nociones sobre ciencia; en este análisis se considera importante realizar las caracterizaciones sobre ciencia, que se han construido a través dela historia; desde concepciones de una ciencia acumulativa hasta la consolidación de la concepción de ciencia actual, en cuyo seno afirmaciones como las siguientes toman sentido:

> Las teorías científicas son totalidades estructuradas.

- Las teorías no son únicas sino que hay teorías rivales que coexisten y se desarmollan a partir de esas contraposiciones.

> La ciencia es una actividad que tiene como objetivo la resolución de problemas.

> La ciencia no sólo se construye a partir de conceptos sino también de sino también de auestiones metodológicas y valorativas.

Con el fin de esclarece las anteriores afirmaciones y consolidar nuestro marco teórico recurriremos a visualizar a partir del siguiente cuadro las caracterizaciones sobre las concepciones de ciencia desde una fuente epistemológica. 
Concepciones sobre la ciencia y su incidencia en la enseĩanza.

La ciencia es entendida como cuerpo de conocimiento acabado, su diseño curricular científico esta basado en secuencia de contenidos conceptuales

CIENCIA

ACUMULATIVA

EMPIRISMO

INDUCTISTA

EL

FALSACIONISMO

DE POPPER

LOS PARADIGMAS DE KUHN

PROGRAMAS DE

INVESTIGACIÓN

DE LAKATOS

TRADICIONES DE

INVESTIGACIÓN

DE LAUDAN

CONCEPCIÓN

ACTUAL DE

CIENCIA definitivos, verdades incuestionables, organizados según la lógica de la materia. Docente dueño del saber y de autoridad indiscutible.

La ciencia se concibe como un aprendizaje de la forma de trabajar de los científicos, su punto de partida es el método científico. El diseño curricular esta basado en los procesos. El aprendizaje se da por desaubrimiento. Los procesos son independientes de los contenidos y la experiencia es la fuente fundamental del conocimiento científico.

La ciencia esta concebida como búsqueda de la "verdad" más que como un medio de desarrollar modelos conceptuales funcionales. Ésta avanza mediante la falsación de hipótesis insostenibles (una Teoría se considera verdadera hasta que se falsee). Se reconoce el carácter evolutivo del conocimiento científico. Esta concepción tiene la virtud de interesarse por el trabajo de los alumnos e introducir en las aulas la importancia de los métodos.

La ciencia se centra en la historia de los descubrimientos científicos, más que en el análisis de los métodos (Conant, 1947). Para Kuhn existen dos tipos de Ciencia: ciencia ordinaria: Resuelve problemas por parte de los científicos.

Ciencia extraordinaria o revolucionaria: Reservada a unos pocos científicos, que son capaces de crear un nuevo paradigma, con mayor poder explicativo.

La ciencia es un hecho colectivo y son fundamentales las características sociológicas de la comunidad cientúfica.

La ciencia esta formada por teorías o programas de investigación que constan de dos componentes: un núcleo central (ideas centrales de la teoría) y un cinturón protector (ideas auxiliares), cuya misión es impedir que el núdeo pueda ser refutado. Todas las teorías, en la medida que no lo explican todo, conviven con anomalías y se pueden incorporar al cinturón protector.

Las tradiciones de investigación son concebidas como un conjunto de teorías en evolución, que no pueden ser evaluadas fuera de su contexto. Éstas poseen reglas de lo que es permitido hacer y no hacer dentro del área de estudio. La función metodológica cuya actividad científica esta encaminada hacia la resolución de problemas, a través de procedimientos (plantear preguntas o problemas y la forma de responderlos y solucionarlos), también se fundamenta en una función ontológica referida a los objetos o fenómenos de estudio de la tradición.

De las posturas de los diferentes Epistemólogos, la ciencia tiene las siguientes características:

$>$ La ciencia se desarrolla en el marco de unas teorías que dirigen la investigación de los científicos.

$>$ Teorías en perpetua revisión y reconstrucción.

$>$ Emisión de hipótesis y su contrastación.

$>$ Una actividad con metodologías no sujetas a reglas fijas, ordenas y universales.

$>\quad$ Una tarea colectiva, que sigue líneas diversas de trabajo aceptadas por la comunidad científica.

> Una actividad impregnada por el momento histórico en el que se desarrolla.

$>\quad$ Una actividad sujeta a intereses sociales y particulares, que a veces es poco objetiva y difícilmente neutra.

\section{Con respecto a nuestras ideas didácticas}

Las siguientes afirmaciones hacen parte de una reflexión en tomo a la enseñanza de las ciencias, que nos permita el puente entre epistemología y didáctica y que además nos posibilite 
acercarnos al análisis sobre la forma como los estudiantes de formación avanzada estructuran los contenidos a enseñar:

\section{- El conocimiento científico suffe transformaciones al convertirse en contenido de enseñanza. \\ - Los contenidos no conforman una sucesión lineal sino una totalidad estructurada. \\ - Los profesores poseen concepciones previas que influyen en su práctica.}

Para apoyar la primera afimmación vamos a recurrir fundamentalmente a Y. Chevallard y su concepto de transposición didáctica, que nos enfatiza en la distancia que separa el conocimiento cientúfico del conocimiento enseñado. Cuando el conocimiento cientúfico se hace objeto de enseñanza, este sufre transformaciones que pueden llegar a ser verdaderas "creaciones didácticas", en términos de este autor.

En la segunda afirmación se enfatiza en la posibilidad de establecer, una especie de paralelismo entre las teorías científicas como totalidades estructuradas y los contenidos de enseñanza. Ya que si un docente en formación es consciente del fenómeno de transposición, desde nuestro punto de vista los contenidos de una asignatura deben conservar la característica de "estructura" propia del conocimiento científico. Es decir, no vemos posible que se pueda trasladar la misma estructura de la ciencia al aula, por lo que si es posible es que pueda hacerse desde su carácter estructural, su carácter de organizarse y de evidenciar las relaciones entre sus conceptos.

De acuerdo con Sánchez, B., et. al., (2000, p. 425) El profesor debe conocer con profundidad; la disciplina, su teoría, leyes y principios, su historia, problemáticas, su práctica metodológica, epistemología y las interacciones CTS, relacionadas con su proceso de construcción.

Situación que debe posibilitar pensar en reflexionar en tomo a lo que deben proporcionar los programas de formación de docentes en donde se debe profundizar en la disciplina y en la reflexión epistemológica. En la organización de contenidos acordes con la concepción de ciencia actual y acorde con las dificultades de aprendizaje de los estudiantes con respecto a la disciplina.

Se necesitan entonces que, el docente tenga criterios daros que le permitan estructurar sus conocimientos y en esa estructuración se debe reflejar la relación entre los contenidos de la disciplina y las situaciones que motiven el aprendizaje de los estudiantes.

Si se miran las diferentes propuesta de estructuración de contenidos según Mellado (1996), se hace relevante el considerar como criterio la práctica de enseñanza que realizan los docentes como una fuente de orientación al respecto.

Las ideas previas de los docentes es otra afirmación de vital importancia, pues la indagación sobre la forma como los docentes estructuran sus contenidos permite evidenciarlas. Creemos que los profesores también tienen ciertas concepciones previas en su práctica docente, que se refieren a diferentes campos: concepciones de la ciencia en general, concepciones de la ciencia que enseñan, concepciones acerca de cómo se produce el conocimiento en los alumnos; seguramente con relación a las concepciones que poseen sobre construcción del conocimiento en general.

Es en esta perspectiva se requiere entonces que se indague sobre la forma como los docentes en formación avanzada estructuran los contenidos, con el fin de ver reflejada la concepción de ciencia y así poder explicitar algunas implicaciones que lleven o no a reformular los programas de formación inicial y avanzada de los docentes.

Dentro de este marco general, el objetivo de esta investigación es realizar los análisis de las concepciones de ciencia de los docentes en formación avanzada implícitas en la forma como 
organizan los contenidos, con ello pretendemos analizar auales son los aiterios que utilizan los docentes a la hora de realizar esa organización y que concepción de ciencia se hace evidente.

\section{Metodología de investigación}

La investigación se realizará bajo un enfoque cualitativo basado en un estudio de casos múltiple. Según Eisenhard (1989), el estudio de casos contemporáneo "es una estrategia de investigación dirigida a comprender las dinámicas presentes en contextos singulares". La cual podría tratarse de un único caso o varios, combinando diferentes métodos para la recogida de la información, con el fin de describir, verificar o generar teoría.

\section{Descripción de la muestra}

Los sujetos con los que se realizó el estudio son 9 estudiantes del programa de educación avanzada: Maestría en Educación con Énfasis en enseñanza de las Ciencias Experimentales de la Universidad de Antioquia; de los cuales 7 imparte la signatura física y 2 la asignatura química. 2 de ellos se desempeñan en educación básica y media, 5 en educación media y universitaria y 2 en educación universitaria solamente. Todos con más de dos años de experiencia docente. 5 tienen una especialización en enseñanza de las ciencias experimentales, 2 otras especializaciones en educación y 2 realizan por primera un programa de educación avanzada. Tenemos entre los sujetos 1 físico, 6 licenciados en física, 1 licenciado en química y 1 químico. Las edades comprendidas están entre los 25-50 años. 3 mujeres y 6 hombres.

\section{Instrumentos para la recogida de la información}

Para la recogida de la información se retomo un o un instrumento escrito de 4 preguntas abiertas de una investigación realizada sobre las SMS, Gess Newsome, Julie y Lederman, $\mathbf{N}$. La estructuración del conocimiento de los maestros en formación de biología como una función de la educación del maestro profesional. Un largo año valorativo. Science Educatión. (1993)

En el cuestionario se pedía que cada estudiante contara cuáles habían sido los temas de física o química que recibieron durante su formación inicial, enumerándolos en orden de importancia. Una segunda pregunta, tenía que ver con los contenidos que ellos consideraban como relevantes o prioritarios para ser enseñados en la educación media. Una tercera situación estaba relacionada con el diseño de un diagrama con los temas que ellos consideran como importantes para ser enseñados. Por último se les pregunta si ellos alguna vez habían reflexionado sobre las preguntas anteriores.

Los criterios de análisis de la información están en relación básicamente con la comparación entre los contenidos que recibieron en su formación inicial con respecto a los que ellos consideran como importantes para ser enseñados. El otro criterio de análisis tiene que ver espećficamente con la concepción de ciencia que se ve reflejada en la forma como cada estudiante estructura los escogidos. Por último se analiza la última pregunta determinando el grado de concientización de los estudiantes de formación avanzada sobre la importancia de reflexionar en la forma como deben organizar los contenidos.

\section{Análisis de los datos}

Para realizar este análisis nos serviremos del cuestionario que los estudiantes respondieron; indagando sobre las ideas que tienen los estudiantes de formación avanzada acerca de cómo 
organizar el contenido de una disciplina a enseñar y con respecto a la forma como les fue enseñado ese contenido.

Cuando se les pide que organicen los contenidos que recibieron durante su formación inicial; de acuerdo al orden de importancia, se observa daramente que la visión de ciencia que se les presentó en cursos anteriores (educación media y formación inicial) no difiere de una visión de ciencia con una postura acumulativa y lineal donde los conocimientos crecen en una línea temporal, ignorando las crisis, las remodelaciones profundas. Esta situación se ve marcada en todos los sujetos. Cuando se les pide que organicen en orden de importancia los contenidos se observa que algunos en su mayoría cambian ese orden.

En la segunda pregunta se les pide que determinen aquellos contenidos que sean prioritarios para ser enseñados se observa que en general todos tienden a reducir o aumentar los contenidos. Sólo 3 de los 9 sujetos cambian de forma radical de los contenidos que consideran prioritarios para ser enseñados, posición que se toma como una visión diferente a la del contexto tradicional.

En la tercera situación se les pide que realicen un diagrama de los contenidos que ellos consideran como prioritarios para ser enseñados se observa gran heterogeneidad al respecto; en todos los diagramas se logra ver que la mayoría de los diagramas responde a un carácter estructural, en donde es posible evidenciar las relaciones entre conceptos. Ya sea desde la enseñanza de un concepto espeć́fico (movimiento) o desde formas de ver como la forma de ver por sistemas y variables. En otros diagramas a pesar de establecer conexiones entre temáticas espećficas no hay daridad en la estructura misma ni criterios daros para el establecimiento de los tipos de relaciones. La mayoría de los diagramas están jerarquizados, sólo uno de ellos tiene una estructura holística. En sus explicaciones algunos dejan ver una intencionalidad diferente a la hora de enseñar, partiendo ya no de temas o conceptos sino a través de explicaciones de fenómenos 0 forma de problemas, estrategia contraria también a lo tradicional.

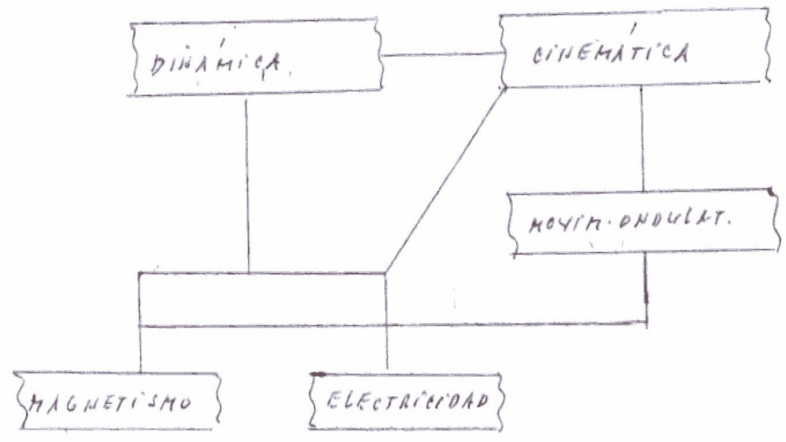

Figura 1. A pesar del diagrama, los contenidos son los mismos, no se deja ver los criterios que permitan tal estructuración.

En la pregunta cuatro; se les pedía que respondieran si antes de aplicarse este cuestionario habían reflexionado sobre las preguntas del cuestionario; a lo cinco de ellos respondieron que sí y aratro no. De los cuatro; dos de ellos ya habían estado cursando programas de educación avanzada.

Finalmente es conveniente adarar que para algunos las preguntas resultaron confusas, situación evidenciada en las respuestas que se obtuvieron, así como se observa en algunos dificultades en el conocimiento de la propia disciplina.

\section{Conclusiones}

En el quehacer educativo, el saber científico se presenta desde una visión didáctica que recibe el nombre de contenido a enseñar que sufre, transformaciones con respecto al conocimiento 
científico inicial, a su vez, los contenidos a enseñar sufren otra transformación cuando el docente los hace objeto efectivo de enseñanza en el salón de dase. Desde este punto observamos que el conocimiento científico, puede sufrir dos transformaciones en donde se corre el riesgo de "deformarlo". Desde la epistemología se permite realizar una vigilancia en donde se realicen una serie de reflexiones con respecto entonces al objeto de estudio que para nuestro caso sería el conocimiento científico, pero además se pueden observar las diferencias entre el conocimiento científico y el saber enseñado.

Desde este punto se hace necesario que en los programas de formación inicial y avanzada se realicen reflexiones sobre la ficción que se vive en la escuela; que consiste en ver en los objetos de enseñanza como copias simplificadas pero fieles de los objetos de la ciencia.

Se hace necesario e importante llamar la atención en que el docente realice a la hora de organizar el conocimiento para ser enseñado un paralelismo entre las teorías científicas como totalidades estructuradas y los contenidos de enseñanza. Pues de acuerdo a lo expresado anteriormente en el marco teórico; los contenidos de una materia deben conservar la característica de "estructura" propia del conocimiento cientúfico; Capacidad de establecer relaciones entre sus conceptos, dejando de lado una mirada aislada de ellos.

Es relevante que los maestros en ejercicio, estudiantes en formación inicial y avanzada tengan en cuenta esta afirmación pues es usual en la enseñanza encontrar el criterio opuesto. Generalmente se observa una postura acumulativa y lineal donde los conocimientos crecen en una línea temporal, ignorando las crisis, las remodelaciones profundas, tal y como se observan en los datos extraídos del cuestionario aplicado al grupo de estudiantes, reflejando esa misma concepción de ciencia; podríamos conduir que solo 2 de ellos tienen una concepción (educación media y formación inicial) de ciencia más cercana a la visión de ciencia holística de Khun.

Creemos que los maestros también tienen ciertas concepciones previas en su práctica docente, que se refieren a diferentes campos: concepciones de la ciencia en general, concepciones de la ciencia que enseñan, concepciones acerca de cómo se produce el conocimiento en los alumnos producto de la relación entre sus concepciones o ideas previas de ciencia y las concepciones de ciencia que les han enseñado, que están muy arraigadas a pesar de ser docentes que cursan programas de formación avanzada y ya haber realizado los cursos de formación inicial de docentes. En otras palabras se puede afirmar que hasta el momento ningún programa a logrado cambios sustanciales al respecto.

Agregamos también, como adelanto de nuestro análisis y con respecto a la epistemología del docente y espećficamente de su concepción de ciencia que: Es implícita, y no es tan argumentada ni fundamenta como la del científico, y que además puede variar según el contexto: inductivismo en algunos casos, relativismo en otros, etc. Esta carencia del maestro para explicitar sus concepciones, este mantenerse en el nivel de los supuestos, no ayuda a configurar una práctica docente eficaz.

En síntesis, consideramos de fundamental importancia el análisis de este punto, ya que la toma de conciencia de esta situación de transposición debería derivar en una práctica en donde el docente de la disciplina vigile epistemológicamente el conocimiento a enseñar". Desde este análisis y reflexión se convoca a que dicha práctica sea liderada a través de los programas de formación de docentes, pues hasta el momento no han dado los cambios sustanciales que al respecto se necesitan para un proceso de enseñanza-aprendizaje eficaz. 


\section{Bibliografía}

Castejón, J.L. (1999). El análisis de tareas en el diseño de la instrucción. En J. Beltrán y C. Genovard (Eds.), Psicología de la instrucción II Áreas aumiaulares (pp. 214-256). Madrid: Síntesis

Eisenhard, K.M (1989). Building Theories from case study research. Academy of Mana-gement Review 14 (4), 532-550

Gess Newsome, J. y Lederman, N. (1993). La estructuración del conocimiento de los maestros en formación de biología como una función de la educación del maestro profesional. Un largo año valorativo. Science Educatión. 77 (1), 25-45.

Sánchez, G. Et. al., (2000). ¿Qué tiene en cuenta los profesores quando seleccionan el contenido de enseñanza? Cambios y dificultades tras un programa de formación. Enseñanza de las Ciencias. 18 (3), 423-437.

Un curríaulo científico para estudiantes de 11 a 14 años. Las fuentes del culmíaulo; La fuente epistemológica. Disponible en: http://uww.oei.gov.co/oeivirt/curnicie/aurri08.html, reauperado el 6 noviembre de 2007 